Revista Latinoamericana de la Papa 21 (1): 73 - 92

http://www.papaslatinas.org/revista.html

ISSN: $1853-4961$

\title{
Comparación de niveles de diversidad genética de papa entre Centros de Agrobiodiversidad y la Colección Nacional de Bolivia
}

\author{
X. Cadima-Fuentes ${ }^{1 / *}$, S. Veramendi ${ }^{1}$, A. Angulo ${ }^{1}$
}

Recibido: 03/01/2017

Aceptado: 10/04/2017

Accesible en línea: Junio 2017

\section{Resumen}

Con el objeto de valorar la riqueza genética de papa cultivada existente en cuatro centros de alta agrobiodiversidad (Norte Potosí, Colomi-Cochabamba, Altiplano Norte-La Paz, Transecto Lago Titicaca-La Paz), se determinó su representatividad respecto a la diversidad total encontrada en la colección nacional de papa boliviana. El análisis de datos moleculares evidenció que la diversidad genética de papa del Norte Potosí es representativa de la diversidad de la colección nacional para las especies $S$. tuberosum subsp. andigena, $S$. stenotomum, S. x juzepczukii y $S . x$ ajanhuiri, porque más del $60 \%$ de los alelos de la colección nacional se hallan representados en este centro. En Colomi se ha encontrado una representatividad importante para las especies $S$. tuberosum subsp. andigena y $S$. stenotomum, porque al menos el 50\% de alelos de la colección nacional están representados en este centro para estas dos especies. El análisis de datos morfológicos muestran que la diversidad genética de papa del transecto del lago (Cachilaya y Coromata) es representativa de la diversidad de la colección nacional para las especies $S$. tuberosum subsp. andigena y S. stenotomum, porque no se encontraron diferencias significativas en la comparación de medias y varianzas en más del $70 \%$ de las variables morfológicas consideradas en este estudio. En las papas del Altiplano norte, la diversidad de S. stenotonum, S. goniocalyx y $S$. $x$ curtilobum representa significativamente lo existente en la colección nacional para estas especies. Las papas de $S$. tuberosum subsp. andigena y $S$. x juzepczukii contienen también una importante variabilidad pero es parcial de lo existente en la colección nacional. Las papas de $S$. $x$ ajanhuiri encontradas en este centro de agrobiodiversidad no representan la variabilidad de la colección nacional. Esta información proporciona mayores argumentos sobre la importancia de la conservación in situ en centros de agrobiodiversidad complementariamente a la conservación ex situ en la colección nacional de germoplasma.

Palabras clave adicionales: Representatividad genética, diversidad, morfológico, molecular.

\footnotetext{
* Dirección de contacto. Correo electrónico: x.cadima@proinpa.org

${ }^{1}$ Fundación PROINPA, Casilla 4285, Cochabamba, Bolivia.
} 


\section{Valuation of genetic diversity of potato from Agrobiodiversity centers}

\section{Summary}

In order to valuate the genetic richness of cultivated potato in four high-agrobiodiversity centers (North Potosí, Colomi-Cochabamba, North Altiplano -La Paz, Lake Titicaca transect-La Paz), their representativity was determined with respect to the total diversity found in the national collection of Bolivian potato. Molecular data analysis showed that the potato genetic diversity of the North Potosí is representative of the diversity of the national collection for $S$. tuberosum subsp. andigena, S. stenotomum, $S$. x juzepczukii and $S$. $x$ ajanhuiri species, because more than $60 \%$ of the alleles of the national collection are represented in this center. In Colomi, an important representativity has been found for the species S. tuberosum subsp. andigena and S. stenotomum, because at least $50 \%$ of the alleles of the national collection are represented in this center for these species. The analysis of the morphological data showed that the potato genetic diversity of the lake transect (Cachilaya and Coromata) is representative of the diversity of the national collection for the species S. tuberosum subsp. andigena and S. stenotomum, because no significant differences were found in the comparison of means and variances in more than $70 \%$ of the morphological variables considered in this study. In the potatoes of the North Altiplano, the diversity of $S$. stenotonum, $S$. goniocalyx and $S$. $x$ curtilobum is representative of what exists in the national collection for these species. The potatoes of $S$. tuberosum subsp. andigena and $S$. x juzepczukii also contain an important variability but is partial of what exists in the national collection. The potatoes of $S$. $x$ ajanhuiri found in this center of agrobiodiversity do not represent the variability of the national collection. This information provides further arguments about the importance of in situ conservation in agrobiodiversity centers complementary to ex situ conservation in the national collection of germplasm.

Additional Key words: Genetic representation, diversity, morphological, molecular.

\section{Introducción}

Bolivia y los otros países andinos de la región comparten el privilegio de ser parte de un centro de origen y domesticación de plantas. Desde muchos siglos atrás, la naturaleza y culturas precolombinas, seleccionaron y domesticaron una gran variedad de recursos fitogenéticos que perduran hasta nuestros días y constituyen la base de la alimentación de las generaciones actuales y futuras (Khoury et al., 2016; Martin \& Sauerborn, 2013; Krapovickas, 2010).

La gran variabilidad de especies sin embargo no es uniforme en toda la franja andina, se concentra más bien en nichos o centros de diversidad con características medio ambientales, sociales y culturales favorables para la conservación de esa rica biodiversidad.

En Bolivia han sido reportados varios centros de diversidad de especies cultivadas, pero muy pocos han sido estudiados y caracterizados (Iriarte et al., 2009; Terrazas et al., 2008; Terrazas et al., 2005; Cadima et al., 2004). Algunos de estos centros mantienen una gran riqueza genética de papa cultivada de diferentes especies. Estos materiales son cultivados por agricultores que han mantenido la diversidad generación tras generación por miles de años. Esta diversidad sin embargo es dinámica y fluctúa por influencia de factores 
socioculturales, pero también está expuesta a una serie de riesgos que pueden afectar negativamente su conservación, tales como la influencia de costumbres externas y por lo tanto la pérdida de tradiciones y costumbres locales, las preferencias de hábito de consumo moderno, el desconocimiento de productos nativos por las nuevas generaciones y la demanda del mercado y preferencia creciente de los consumidores por productos de origen industrial (FAO, 2017a; Eyzaguirre-Rodríguez, 2015). Además de factores bióticos, tales como enfermedades y plagas que podrían provocar eventualmente erosión genética debido a un incremento alarmante de estos problemas exacerbados por el cambio climático y los peligros de la globalización que inducen al aumento de plagas y enfermedades transfronterizas (FAO, 2017a y b; Castillo \& Plata, 2016).

Es importante tener una mayor certeza sobre la diversidad presente en los centros de agrobiodiversidad, es decir valorar la riqueza genética existente en estos centros y estimar cuál es su representatividad respecto a la diversidad total. Esta información proporcionará mayores argumentos sobre la importancia de la conservación in situ en centros de agrobiodiversidad y/o la necesidad de colecciones ex situ.

Se reconoce que para medir la diversidad genética total de una colección, idealmente se deberían considerar los diferentes tipos de caracterización (morfológica + evaluación + molecular) (Franco \& Hidalgo, 2003). Sin embargo en la práctica, difícilmente los bancos de germoplasma cuentan con los recursos necesarios para medir todos los niveles de variabilidad (fenotípica, evaluativa y molecular), más aún cuando se tratan de colecciones grandes ex situ o colecciones in situ de varias regiones. Por ello, los análisis más usados y disponibles para medir la variabilidad genética siguen siendo las que se expresan en características visibles especialmente la caracterización morfológica y la evaluación (Jarvis et al., 2016).

En el presente trabajo se ha hecho el ejercicio de la valoración de la diversidad genética tomando como estudio de caso el cultivo de la papa. Se identificaron cuatro centros de agrobiodiversidad (Norte Potosí, Colomi-Cochabamba, Altiplano Norte-La Paz, Transecto Lago TiticacaLa Paz) en Bolivia, donde la papa es el principal cultivo en términos de diversidad e importancia sociocultural y económica para las comunidades presentes en estos centros.

Se tomó también en cuenta información previa desarrollada por la Fundación PROINPA sobre estudios de diversidad con datos morfológicos y moleculares de la colección nacional que comprende siete especies de papa cultivada de acuerdo a la clasificación taxonómica convencional de Ochoa (1990) y Hawkes \& Hjerting (1989) (S. tuberosum subsp. andigena, $S$. phureja, S. goniocalyx, S. stenotomum, $S$. $x$ ajanhuiri, $S . \quad x$ curtilobum y $S . \quad x$ juzepczukii) de toda la región andina de Bolivia, principalmente de los departamentos de La Paz, Oruro, Potosí, Cochabamba y Chuquisaca, en menor proporción de Tarija y escasamente de los valles mesotérmicos de Santa Cruz.

Con estas consideraciones, se propuso estimar la diversidad genética de papa cultivada de cuatro centros de agrobiodiversidad, comparando la diversidad encontrada en cada centro, en base a información disponible molecular y morfológica, con la diversidad de la colección nacional. La información molecular se obtuvo de marcadores microsatélites y la información 
morfológica se tomó en cuenta de caracteres que expresan pequeña variación con el ambiente, como los datos de tubérculo, flor y planta. No así datos de evaluación agronómica porque varían considerablemente con el ambiente (Jarvis et al., 2016).

\section{Materiales y métodos}

Material biológico
En la Tabla 1 se detalla el número de cultivares o accesiones de papa que fueron considerados en el estudio provenientes de cuatro centros de agrobiodiversidad del área andina de Bolivia: Colomi (Cochabamba), Norte Potosí (Potosí), Cachilaya y Coromata del transecto del Lago Titicaca (La Paz), y la región del Altiplano Norte (La Paz).

Tabla 1. Número de cultivares por especie de papa cultivada de cada Centro de Agrobiodiversidad considerados en el estudio

\begin{tabular}{|c|c|c|c|c|c|}
\hline \multirow{3}{*}{$\begin{array}{l}\text { Especie de papa } \\
\text { cultivada }\end{array}$} & \multicolumn{5}{|c|}{ Centros de Agrobiodiversidad } \\
\hline & \multirow{2}{*}{ Norte Potosí } & \multirow{2}{*}{ Colomi* } & \multicolumn{2}{|c|}{ Transecto Lago $* *$} & \multirow{2}{*}{ Altiplano Norte } \\
\hline & & & & Coromata & \\
\hline \multicolumn{6}{|l|}{ Solanum } \\
\hline $\begin{array}{l}\text { tuberosum subsp. } \\
\text { andigena }\end{array}$ & 131 & 31 & 34 & 24 & 99 \\
\hline S. x ajanhuiri & 21 & & & & 4 \\
\hline S. x juzepczukii & 49 & 2 & & & 14 \\
\hline S. stenotomum & 109 & 9 & 13 & 17 & 15 \\
\hline S. $x$ curtilobum & 5 & & & & 3 \\
\hline S. goniocalyx & 5 & & & & 5 \\
\hline Total accesiones & 320 & 52 & 8 & 8 & 140 \\
\hline \multicolumn{6}{|c|}{$\begin{array}{l}\text { *En Colomi también se encontró un número importante de cultivares de } S \text {. phureja }(19) \\
\text { pero no se cuenta con datos de caracterización molecular ni morfológica de estos materiales } \\
\text { en la colección de Colomi ni en la colección nacional. } \\
\text { ** En las comunidades del Transecto del Lago (Cachilaya y Coromata) también fueron } \\
\text { encontradas otras especies como } S . x \text { juzepczukii, } S . x \text { curtilobum, } S \text {. goniocalyx y } S . x \\
\text { ajanhuiri, pero con un número muy reducido de cultivares }(\leq 2) \text { por lo que no fueron }\end{array}$} \\
\hline
\end{tabular}




\section{Análisis Molecular}

La extracción de ADN genómico de las accesiones de papa se realizó mediante el protocolo de CTAB 2X (hexadecil bromuro de trimetil amonio) de Doyle y Doyle (1990), con algunas modificaciones.

La cuantificación de ADN genómico se realizó por el método de electroforesis en gel de agarosa al $1 \%$ y se visualizaron las bandas con bromuro de etidio.

La amplificación de fragmentos se realizó en un termociclador GeneAmp PCR System 9700 (Applied Biosystems, USA).

Se eligieron como marcadores moleculares para este estudio a los microsatélites (SSR) debido a sus características de elevado polimorfismo, codominancia y multialelismo (He et al. 2003), y porque solo se necesita una pequeña cantidad de ADN para la amplificación, siendo ésta de 5 - 50 ng por reacción (Torrez \& Moreno 2001). En la Tabla 2 se muestra la lista de los microsatélites utilizados en la caracterización de la diversidad genética de papa cultivada de dos centros de agrobiodiversidad (Norte Potosí y Colomi). Estos microsatélites fueron elegidos por Guislain et al. (2009) como los más informativos para el genotipado de cultivares de papa. 
Tabla 2. Características de los microsatélites utilizados en la caracterización de la diversidad genética del material proveniente del Norte Potosí y de Colomi.

\begin{tabular}{|c|c|c|c|}
\hline Microsatélite & Motivo Repetido & Secuencia de iniciador & Th ${ }^{\circ} \mathbf{C}$ \\
\hline \multirow[t]{2}{*}{$\overline{\text { STG } 0001}$} & $(\mathrm{CT}) \mathrm{n}$ & F AGCCAACATTTGTACCCCT & 58.0 \\
\hline & & R ACCCCCACTTGCCATATTTT & 52.0 \\
\hline \multirow{2}{*}{ STG 0010} & (TG)n & F CGATCTCTGCTTTGCAGGTA & 60.0 \\
\hline & & R GTTCATCACTACCGCCGACT & 55.0 \\
\hline \multirow{2}{*}{ STG 0016} & $(\mathrm{AGA}) \mathrm{n}$ & F AGCTGCTCAGCATCAAGAGA & 55.0 \\
\hline & & R ACCACCTCAGGCACTTCATC & 53.0 \\
\hline \multirow[t]{2}{*}{ STG 0025} & $(\mathrm{AAAC}) \mathrm{n}$ & F TGGAATCCGAATTACGCTCT & 56.0 \\
\hline & & R AGGTTTTACCACTCGGGCTT & 55.0 \\
\hline \multirow[t]{2}{*}{ STI 0001} & $(\mathrm{AAT}) \mathrm{nr}$ & F CAGCAAAATCAGAACCCGAT & 60.0 \\
\hline & & R GGATCATCAAATTCACCGCT & 55.0 \\
\hline \multirow[t]{2}{*}{ STI 0004} & $(\mathrm{AAG}) \mathrm{n}$ & F GCTGCTAAACACTCAAGCAGAA & 60.0 \\
\hline & & R CAACTACAAGATTCCATCCACAG & 55.0 \\
\hline \multirow[t]{2}{*}{ STI 0012} & $(\mathrm{ATT}) \mathrm{n}$ & F GAAGCGACTTCCAAAATCAGA & 56.0 \\
\hline & & R AAAGGGAGGAATAGAAACCAAAA & 55.0 \\
\hline \multirow[t]{2}{*}{ STI 0014} & (TGG)n (AGG)n & F AGAAACTGAGTTGTGTTTGGGA & 54.0 \\
\hline & & R TCAACAGTCTCAGAAAACCCTCT & 55.0 \\
\hline \multirow[t]{2}{*}{ STI 0030} & (ATT)n & F TTGACCCTCCAACTATAGATTCTTC & 58.0 \\
\hline & & R TGACAACTTTAAAGCATATGTCAGC & 60.0 \\
\hline \multirow[t]{2}{*}{ STI 0031} & (TCA)n & F AGGCGCACTTTAACTTCCAC & 60.0 \\
\hline & & R CGGAACAAATTGCTCTGATG & 54.0 \\
\hline \multirow[t]{2}{*}{ STI 0032} & (GGA)nr & F TGGGAAGAATCCTGAAATGG & 61.0 \\
\hline & & $\mathrm{R}$ TGCTCTACCAATTAACGGCA & 60.0 \\
\hline \multirow[t]{2}{*}{ STI 0033} & $(\mathrm{AGG}) \mathrm{n}$ & F TGAGGGTTTTCAGAAAGGGA & 61.0 \\
\hline & & R CATCCTTGCAACAACCTCCT & 60.0 \\
\hline \multirow[t]{2}{*}{ STM 0019} & $(\mathrm{AT}) 7(\mathrm{GT}) 10(\mathrm{AT}) 4$ & F AATAGGTGTACTGACTCTCAATG & 52.8 \\
\hline & (GT)5 (GC)4(GT)4 & R TTGAAGTAAAAGTCCTAGTAGTG & 52.6 \\
\hline \multirow[t]{2}{*}{ STM 0037} & $(\mathrm{TC}) \mathrm{n}(\mathrm{AC}) \mathrm{n}$ & F AATTTAACTTAGAAGATTAGTCTC & 52.0 \\
\hline & AA...(AC)n(AT)n & R ATTTGGTTGGGTATGATA & 53.0 \\
\hline \multirow[t]{2}{*}{ STM 1052} & $(\mathrm{AT}) \mathrm{n}$ GT(AT)n(GT)n & F CAATTTCGTTTTTTCATGTGACAC & 50.0 \\
\hline & & R ATGGCGTAATTTGATTTAATACGTAA & 52.0 \\
\hline \multirow[t]{2}{*}{ STM 1053} & (TA)n (ATC)n & F TCTCCCCATCTTAATGTTTC & 53.0 \\
\hline & & R CAACACAGCATSCAGATCATC & 53.0 \\
\hline \multirow[t]{2}{*}{ STM1064 } & (TA)n (TG) GT & F GTTCTTTTGGTGGTTTTCCT & 55.0 \\
\hline & (TG)n & $\mathrm{R}$ TTATTTCTCTGTTGTTGCTG & 55.0 \\
\hline \multirow[t]{2}{*}{ STM 1104} & (TCT)n & F TGATTCTCTTGCCTACTGTAATCG & 53.0 \\
\hline & & R CAAAGTGGTGTGAAGCTGTGA & 57.0 \\
\hline \multirow[t]{2}{*}{ STM 5114} & $(\mathrm{ACC}) \mathrm{n}$ & F AATGGCTCTCTCTGTATGCT & 60.0 \\
\hline & & R GCTGTCCCAACTATCTTTGA & 57.0 \\
\hline \multirow[t]{2}{*}{ STM 1106} & (ATT)n & F TCCAGCTGATTGGTTAGGTTG & 51.0 \\
\hline & & R ATGCGAATCTACTCGTCATGG & 55.0 \\
\hline \multirow[t]{2}{*}{ STM 5127} & (TCT)n & F TTCAAGAATAGGCAAAACCA & 55.0 \\
\hline & & R CTTTTTCTGACTGAGTTGCCTC & 60.0 \\
\hline \multirow[t]{2}{*}{ STPoAc58 } & (TA)n & F TTGATGAAAGGAATGCAGCTTGTG & - \\
\hline & & R ACGTTAAAGAAGTGAGAGTACGAC & 57.0 \\
\hline
\end{tabular}

Para la evaluación de las papas del Norte Potosí se utilizaron 20 microsatélites (todos de la lista de la Tabla 2 menos el 
STI 0031 y el STM 5127) y para las papas de Colomi se utilizaron los 22 microsatélites de la lista.

Evaluación de la diversidad genética y estructura poblacional en base a datos moleculares

Los perfiles moleculares producto de la amplificación de microsatélites fueron transformados a matrices binarias (1 presencia y 0 ausencia), con las que se generó la matriz de distancias utilizando el coeficiente de similitud de Jaccard con el programa NTSyS PC-2.10 (Rohlf, 2004). Se realizó el cálculo de los índices de diversidad, como el índice de contenido polimórfico (PIC) y las frecuencias alélicas. La frecuencia alélica se define como una medida de la presencia continua $\mathrm{o}$ asidua de un alelo en una población, o de la proporción que representa todos los alelos de un gen (de Vicente \& Fulton, 2004). El Índice de Contenido Polimórfico (PIC por sus siglas en inglés) fue obtenido con la fórmula de Botstein et al. (1980) y evalúa cuán informativo en términos de polimorfismo es un marcador en la población de acuerdo a las frecuencias de los alelos y sus valores oscilan entre 0 y 1 .

\section{Análisis morfológico}

Se utilizaron descriptores morfológicos del Centro Internacional de la Papa (CIP) (Gómez, 2000) para describir la diversidad de papa de los centros del transecto del Lago (Cachilaya y Coromata) y del Altiplano Norte, en base a sus caracteres de tubérculo, flor, planta y hábito de crecimiento en particular (Tabla 3). 
Tabla 3. Detalle de los descriptores morfológicos utilizados en la caracterización de la diversidad genética del material proveniente del Transecto del Lago (Cachilaya y Coromata) y Altiplano Norte.

\begin{tabular}{cll}
\hline $\mathbf{N}^{\mathbf{0}}$ & & \multicolumn{1}{c}{ Caracteres morfológicos } \\
\hline 1 & HPL*++ & Habito de crecimiento planta \\
2 & TDS*++ & Tipo de disección \\
3 & CTL++ & Color del tallo \\
4 & GFL++ & Grado de floración \\
5 & FCL++ & Forma de la corola \\
6 & CPF++ & Color predominante de la flor \\
7 & INT++ & Intensidad del color predominante de la flor \\
8 & CSF++ & Color secundario de la flor \\
9 & DCS++ & Distribución color secundario de la flor \\
10 & CLZ & Color del cáliz \\
11 & CBY & Color de la baya \\
12 & FBY & Forma de la baya \\
13 & CPPL*++ & Color predominante de la piel del tubérculo \\
14 & INTC*++ & Intensidad del color predominante de la piel del tubérculo \\
15 & CSP*++ & Color secundario de la piel del tubérculo \\
16 & DSCPL*++ & Distribución del color secundario de la piel del tubérculo \\
17 & CPP++ & Color predominante de la pulpa del tubérculo \\
18 & CSPL++ & Color secundario de la pulpa del tubérculo \\
19 & DCSP++ & Distribución del color secundario de la pulpa del tubérculo \\
20 & FGR*++ & Forma general del tubérculo \\
21 & FRA*++ & Forma rara del tubérculo \\
22 & P0J*++ & Profundidad de los ojos \\
\hline
\end{tabular}

Para la evaluación de las papas de Cachilaya se utilizaron los 22 descriptores de la lista de la Tabla 3. Los descriptores con * fueron utilizados para la caracterización de las papas de Coromata. Los descriptores con ++ fueron utilizados para la caracterización de las papas del Altiplano Norte.

\section{Comparación de la diversidad encontrada en centros de agrobiodiversidad de papa con la diversidad de la colección nacional}

Los resultados de la evaluación de la diversidad genética obtenidos mediante datos moleculares y morfológicos para las colecciones de papa de cada centro de agrobiodiversidad fueron posteriormente comparados con datos similares de la colección nacional para determinar la representatividad de la diversidad en cada centro. Los datos moleculares que fueron comparados entre colecciones fueron las variantes alélicas, el rango de pares de bases y el índice de contenido polimórfico (PIC). En el caso de los datos morfológicos se compararon las varianzas utilizando $\mathrm{Xi}$ cuadrado de Barlett y la comparación de medias se hizo mediante la distribución $\mathrm{F}$ de Snedecor (Snedecor \& Cochran, 1989).

\section{Resultados y discusión}

\section{Caracterización de la diversidad genética de papa del Norte Potosí}

Los resultados encontrados sobre el número de las variantes alélicas y el rango de tamaño de alelos (en pb) para cada una de las especies de papa del Norte Potosí se encuentran dentro de los valores del rango de tamaño de alelos reportados por Cadima et al. (2013) en la evaluación de la colección total de papa de Bolivia (Tabla 4). 
Tabla 4. Número de accesiones, variantes alélicas y rango en pares de bases por especie de las papas del Norte Potosí y de la colección nacional.

\begin{tabular}{lccc|ccc}
\hline & \multicolumn{3}{c|}{ Colección Norte Potosí } & \multicolumn{3}{c}{ Colección Bolivia } \\
\hline \multicolumn{1}{c}{ Especie/ploidia* } & $\begin{array}{c}\text { No. de } \\
\text { accesiones }\end{array}$ & $\begin{array}{c}\text { No. de } \\
\text { variantes } \\
\text { alélicas }\end{array}$ & $\begin{array}{c}\text { Rango en } \\
\text { pares de } \\
\text { bases }\end{array}$ & $\begin{array}{c}\text { No. de } \\
\text { accesiones }\end{array}$ & $\begin{array}{c}\text { No. de } \\
\text { variantes } \\
\text { alélicas }\end{array}$ & $\begin{array}{c}\text { Rango pares } \\
\text { de bases }\end{array}$ \\
\hline $\begin{array}{l}\text { Solanum tuberosum subsp. } \\
\text { andigena }(4 \mathrm{x})\end{array}$ & 131 & 252 & $129-303$ & 911 & 287 & $75-318$ \\
Solanum x ajanhuiri $(2 \mathrm{x})$ & 21 & 71 & $134-297$ & 56 & 119 & $79-311$ \\
Solanum x juzepczukii & 49 & 116 & $134-303$ & 116 & 186 & $83-318$ \\
$\begin{array}{l}\text { (3x) } \\
\text { Solanum stenotomum }(2 \mathrm{x})\end{array}$ & 109 & 188 & $129-303$ & 228 & 241 & $87-318$ \\
Solanum x curtilobum $(5 x)$ & 5 & 50 & $83-303$ & 78 & 160 & $86-318$ \\
Solanum goniocalyx $(2 x)$ & 5 & 40 & $79-303$ & 5 & 150 & $79-318$ \\
\hline
\end{tabular}

* 2x: Diploide, 3x: Triploide, 4x: Tetraploide, 5x: Pentaploide.

La especie que presentó el mayor número de alelos fue $S$. tuberosum subsp. andigena con 252 alelos los cuales corresponden al $88 \%$ de los alelos encontrados en la colección nacional para esta misma especie. En S. stenotomum, S. x juzepczukii y S. $x$ ajanhuiri se encontraron 188, 116 y 71 alelos respectivamente, que corresponden al 78, 62 y $60 \%$ de los alelos encontrados en la colección nacional para estas mismas especies respectivamente. Menor número de alelos fueron encontrados en $S . \quad x$ curtilobum y $S$. goniocalyx, lo cual coincide con el menor número de cultivares de estas especies en el Norte Potosí. Sin embargo a pesar del bajo número de cultivares, éstas reportaron un 31 y $27 \%$ de los alelos de la colección nacional.

La mayor cantidad de alelos detectados en $S$. tuberosum subsp. andigena, tiene relación con el mayor número de accesiones analizadas $(n=131)$ en comparación con las otras especies $S$. stenotomum (109), S. x juzepczukii (49), $S$. $x$ ajanhuiri (21), $S$. x curtilobum (5) y $S$. goniocalyx (5).

Resalta también que $S$. stenotomum a pesar de ser una especie diploide $(2 \mathrm{x})$ presenta una alta diversidad genética, mucho mayor a las otras especies (S. $x$ ajanhuiri $2 x$ y $S . x$ juzepczukii 3x). Lo cual coincide con los resultados obtenidos en el estudio de la colección total de papa boliviana donde los cultivares de esta especie están en mayor número (Cadima et al., 2013) y también son los más abundantes y diversos en la región andina (de Haan et al., 2010).

El Índice de Contenido Polimórfico (PIC) evalúa la informatividad de un marcador en la población de acuerdo a las frecuencias de los alelos (Aranguren Mendez et al., 2001). Como se aprecia en la Tabla 5 el valor del PIC para un mismo microsatélite puede variar mucho entre especies. Los valores por microsatélite estuvieron en un rango de 0,13 a 0,85 para la colección de papa del Norte Potosí y en cada especie el mayor valor de PIC se tuvo en un microsatélite diferente. En líneas generales los valores PIC por marcador en la colección del Norte Potosí fueron menores que los encontrados en la colección nacional, y los valores PIC de la colección nacional resultaron ser ligeramente menores que los valores encontrados en análisis de la colección de papa cultivada del Centro Internacional de la Papa (CIP) (Ghislain et al., 2009). Estas diferencias pueden deberse a dos factores: 1) número de cultivares analizados (caso colección in situ con menos accesiones), y 2) rango geográfico considerado (caso 
colección del CIP con cultivares de varios países). Similar situación se detectó también en los valores PIC de los mismos marcadores microsatélites utilizados en cultivares locales de papa de las islas Canarias (Ruiz de Galarreta et al., 2007).

Considerando los valores de PIC promedios por especie, se observa que $S$. tuberosum subsp. andigena registró el mayor valor de PIC $(0,71)$, seguido por $S$. stenotomum $(0,65), S$. x juzepczukii $(0,55)$, y $S$. $x$ ajanhuiri $(0,42)$. Estos valores son similares a los encontrados para las mismas especies en la colección nacional
(Cadima et al., 2013; Tabla 5). En cambio para el caso de $S$. $x$ curtilobum y $S$. goniocalyx, los valores PIC promedio $(0.42$ y 0.25 respectivamente) son menores de los valores para estas mismas especies de la colección nacional. Esto puede deberse al número reducido de cultivares de $S . x$ curtilobum evaluados del Norte Potosí y la poca variabilidad genética común en esta especie, y en el caso de $S$. goniocalyx los resultados muestran que los cultivares de esta especie son similares genéticamente en el Norte Potosí.

Tabla 5. Valores del PIC para los 20 microsatélites utilizados en la evaluación de las papas del Norte Potosí y de la colección nacional.

Valor de PIC por especie

\begin{tabular}{|c|c|c|c|c|c|c|c|c|c|c|c|c|c|c|}
\hline \multirow[t]{2}{*}{ *SSR } & \multicolumn{7}{|c|}{ Colección Norte Potosí } & \multicolumn{7}{|c|}{ Colección Bolivia } \\
\hline & $\overline{\mathrm{ADG}}$ & $\overline{\text { AJH }}$ & JUZ & $S$ & CUR & GON & Promedio & ADG & AJH & $\mathbf{J U Z}$ & STN & CUR & GON & Promedio \\
\hline STG0001 & 0,62 & 0,38 & 0,38 & 0,55 & - & 0,35 & 0,38 & 0,67 & 0,55 & 0,52 & 0,73 & 0,69 & 0,76 & 0,65 \\
\hline STG0010 & 0,71 & 0,64 & 0,60 & 0,69 & 0,38 & - & 0,50 & 0,79 & 0,72 & 0,67 & 0,73 & 0,78 & 0,68 & 0,73 \\
\hline STG0016 & \begin{tabular}{|l|}
0,84 \\
\end{tabular} & 0,48 & 0,59 & 0,76 & 0,67 & - & 0,56 & 0,81 & 0,75 & 0,55 & 0,73 & $\mathbf{0 , 8 1}$ & 0,74 & 0,73 \\
\hline STG0025 & 0,77 & 0,51 & 0,59 & 0,67 & 0,35 & 0,38 & 0,54 & 0,50 & 0,57 & 0,38 & 0,41 & 0,41 & - & 0,38 \\
\hline STI0001 & \begin{tabular}{|l|}
$\mathbf{0 , 8 4}$ \\
\end{tabular} & 0,59 & 0,64 & $\mathbf{0 , 8 5}$ & 0,70 & - & 0,60 & 0,54 & 0,39 & 0,25 & 0,49 & 0,70 & 0,36 & 0,46 \\
\hline STI0004 & 0,45 & 0,28 & 0,38 & 0,15 & 0,38 & - & 0,27 & 0,67 & 0,52 & 0,72 & 0,36 & 0,63 & 0,19 & 0,52 \\
\hline STI0012 & 0,79 & 0,65 & 0,68 & 0,70 & - & 0,38 & 0,53 & 0,72 & 0,44 & 0,66 & 0,58 & 0,74 & 0,37 & 0,59 \\
\hline STI0014 & 0,75 & 0,59 & 0,67 & 0,68 & 0,70 & 0,38 & 0,63 & 0,53 & 0,39 & 0,61 & 0,50 & 0,50 & 0,46 & 0,50 \\
\hline STI0030 & 0,73 & 0,28 & 0,59 & 0,79 & 0,70 & 0,56 & 0,61 & 0,81 & 0,24 & 0,73 & 0,79 & 0,51 & $\mathbf{0 , 7 7}$ & 0,64 \\
\hline STI0032 & 0,83 & 0,51 & 0,70 & 0,77 & 0,59 & 0,74 & 0,69 & 0,59 & 0,70 & 0,71 & 0,71 & 0,68 & 0,63 & 0,67 \\
\hline STI0033 & 0,70 & 0,37 & 0,43 & 0,56 & 0,35 & - & 0,40 & 0,62 & 0,13 & 0,39 & 0,44 & 0,41 & - & 0,33 \\
\hline STM0019 & 0,70 & 0,27 & 0,65 & 0,78 & 0,38 & - & 0,46 & 0,73 & 0,24 & 0,62 & 0,70 & 0,33 & - & 0,44 \\
\hline STM0037 & \begin{tabular}{|l|}
$\mathbf{0 , 8 4}$ \\
\end{tabular} & 0,37 & 0,54 & 0,71 & 0,38 & - & 0,47 & $\mathbf{0 , 8 7}$ & 0,78 & 0,53 & 0,79 & 0,73 & 0,60 & 0,72 \\
\hline STM1052 & 0,77 & 0,38 & 0,57 & 0,72 & 0,38 & - & 0,47 & 0,77 & 0,55 & 0,39 & \begin{tabular}{|l|}
0,85 \\
\end{tabular} & 0,61 & 0,19 & 0,56 \\
\hline STM1053 & 0,45 & 0,59 & 0,13 & 0,69 & - & - & 0,31 & 0,57 & 0,47 & 0,51 & 0,61 & 0,51 & 0,46 & 0,52 \\
\hline STM1064 & 0,44 & 0,37 & 0,59 & 0,34 & 0,59 & - & 0,39 & 0,52 & 0,53 & 0,61 & 0,45 & 0,60 & 0,19 & 0,48 \\
\hline STM1104 & 0,83 & 0,50 & $\mathbf{0 , 7 3}$ & 0,77 & 0,70 & 0,73 & 0,71 & 0,85 & 0,54 & 0,62 & 0,83 & 0,57 & 0,75 & 0,69 \\
\hline STM5114 & 0,59 & 0,37 & 0,63 & 0,49 & 0,70 & 0,38 & 0,53 & 0,78 & $\mathbf{0 , 8 0}$ & 0,64 & 0,70 & $\mathbf{0 , 8 1}$ & 0,70 & 0,74 \\
\hline STM1106 & 0,79 & 0,00 & 0,28 & 0,72 & 0,38 & 0,36 & 0,42 & 0,58 & 0,07 & 0,17 & 0,82 & 0,05 & 0,75 & 0,41 \\
\hline STPoAc58 & 0,80 & 0,37 & 0,69 & 0,58 & 0,38 & 0,00 & 0,47 & 0,62 & 0,40 & 0,65 & 0,56 & 0,50 & 0,34 & 0,51 \\
\hline Promedio & 0,71 & 0,42 & 0,55 & 0,65 & 0,42 & 0,25 & & 0,68 & 0,48 & 0,52 & 0,64 & 0,58 & 0,45 & \\
\hline
\end{tabular}


*SSR: Simple Sequence Repeats (Marcador microsatélite), ADG: Solanum tuberosum subsp. andigena; AJH: Solanum x ajanhuiri; JUZ: Solanum x juzepczukii; STN: Solanum stenotomum. Solanum x curtilobum, Solanum goniocalyx. Los valores de PIC enmarcados corresponden a los valores mayores para cada marcador y especie. 
Tabla 6. Número de accesiones, variantes alélicas y rango en pares de bases por especie de las papas de Colomi y de la colección nacional.

\begin{tabular}{|c|c|c|c|c|c|c|}
\hline \multicolumn{4}{|c|}{ Colección Colomi } & \multicolumn{3}{|c|}{ Colección Bolivia } \\
\hline Especie/ploidia* & $\begin{array}{l}\text { No. de } \\
\text { accesiones }\end{array}$ & $\begin{array}{c}\text { No. de } \\
\text { variante } \\
\text { S alélicas }\end{array}$ & $\begin{array}{c}\text { Rango en } \\
\text { pares de } \\
\text { bases }\end{array}$ & $\begin{array}{c}\text { No. de } \\
\text { accesiones }\end{array}$ & $\begin{array}{c}\text { No. de } \\
\text { variantes } \\
\text { alélicas }\end{array}$ & $\begin{array}{c}\text { Rango en } \\
\text { pares de } \\
\text { bases }\end{array}$ \\
\hline $\begin{array}{l}\text { Solanum tuberosum subsp. } \\
\text { andigena }(4 \mathrm{x})\end{array}$ & 31 & 163 & $89-318$ & 911 & 287 & $75-318$ \\
\hline Solanum stenotomum $(2 \mathrm{x})$ & 19 & 118 & $78-291$ & 228 & 241 & $87-318$ \\
\hline $\begin{array}{l}\text { Solanum x juzepczukii } \\
(3 \mathrm{x})\end{array}$ & 2 & 47 & $86-302$ & 116 & 186 & 83- 318 \\
\hline
\end{tabular}

* 2x: Diploide, 3x: Triploide, 4x: Tetraploide.

Como se esperaba, la mayor diversidad observada fue en la especie $S$. tuberosum subsp. andigena, debido a que los cultivares de esta especie son los más abundantes y diversos en la región andina (de Haan et al., 2010; Guislain et al., 2009). El número de alelos encontrados en esta especie para Colomi representa el 57\% de los encontrados en la colección nacional. El número de alelos encontrados en S. stenotomum representa el $49 \%$ de la colección nacional, y el número de alelos en $S$. x juzepczukii representa el $25 \%$ de la colección nacional. Es importante resaltar que estos porcentajes fueron hallados en un bajo número de accesiones provenientes de Colomi: 31 accesiones de S. tuberosum subsp. andigena representa solo el 3.4\% de la colección nacional, 19 de $S$. stenotomum representa el $8 \%$ y 2 de $S$. x juzepczukii representa el $1.7 \%$ de la colección nacional.

Como fue mencionado antes, el mayor número de alelos encontrados en una especie tiene relación con el mayor número de accesiones analizadas; es el caso de las 31 accesiones de $S$. tuberosum subsp. andigena analizadas de Colomi en comparación con las otras especies (19 para S. stenotomum y 2 para $S . x$ juzepczukii). Cuanto más grande es la población aumenta más la probabilidad de encontrar nuevos alelos con frecuencias bajas (Pérez 2004.) Estos resultados son coincidentes a los reportados por Cadima et al. (2013).

El valor del PIC para un mismo microsatélite varía mucho entre especies como se observa en la Tabla 7. 
Tabla 7.Valores del PIC para 22 microsatélites utilizados en las especies de papa de Colomi y de la colección nacional

\begin{tabular}{|c|c|c|c|c|c|c|c|c|}
\hline \multirow{3}{*}{ SSR } & \multicolumn{8}{|c|}{ Valor de PIC por Especie } \\
\hline & \multicolumn{4}{|c|}{ Colección Colomi } & \multicolumn{4}{|c|}{ Colección Bolivia } \\
\hline & ADG & STN & JUZ & Promedio & ADG & STN & JUZ & Promedio \\
\hline STG0001 & 0,72 & 0,62 & 0,38 & 0,67 & 0,67 & 0,73 & 0,52 & 0,7 \\
\hline STG0010 & 0,76 & 0,72 & 0,59 & 0,74 & 0,79 & 0,73 & 0,67 & 0,76 \\
\hline STG0016 & 0,79 & 0,7 & 0,38 & 0,75 & 0,81 & 0,73 & 0,55 & 0,77 \\
\hline STG0025 & 0,52 & 0,5 & 0,38 & 0,51 & 0,5 & 0,41 & 0,38 & 0,46 \\
\hline STI0001 & 0,53 & 0,45 & 0,00 & 0,49 & 0,54 & 0,49 & 0,25 & 0,52 \\
\hline STI0004 & 0,61 & 0,28 & 0,59 & 0,45 & 0,67 & 0,36 & 0,72 & 0,52 \\
\hline STI0012 & 0,69 & 0,52 & 0,67 & 0,6 & 0,72 & 0,58 & 0,66 & 0,65 \\
\hline STI0014 & 0,5 & 0,41 & 0,59 & 0,45 & 0,53 & 0,5 & 0,61 & 0,52 \\
\hline STI0030 & 0,81 & 0,72 & 0,55 & 0,76 & 0,81 & 0,79 & 0,73 & 0,8 \\
\hline STI0031 & 0,61 & 0,36 & - & 0,48 & 0,69 & 0,54 & 0 & 0,62 \\
\hline STI0032 & 0,58 & 0,7 & 0,38 & 0,64 & 0,59 & 0,71 & 0,71 & 0,65 \\
\hline STI0033 & 0,58 & 0,45 & 0,38 & 0,51 & 0,62 & 0,44 & 0,39 & 0,53 \\
\hline STM0019 & 0,66 & 0,47 & 0,59 & 0,57 & 0,73 & 0,7 & 0,62 & 0,72 \\
\hline STM0037 & 0,83 & 0,72 & 0,38 & 0,77 & 0,87 & 0,79 & 0,53 & 0,83 \\
\hline STM1052 & 0,77 & 0,74 & 0,38 & 0,75 & 0,77 & 0,85 & 0,39 & 0,81 \\
\hline STM1053 & 0,51 & 0,4 & 0,38 & 0,45 & 0,57 & 0,61 & 0,51 & 0,59 \\
\hline STM1064 & 0,45 & 0,31 & 0,70 & 0,38 & 0,52 & 0,45 & 0,61 & 0,49 \\
\hline STM1104 & 0,84 & 0,77 & 0,00 & 0,81 & 0,85 & 0,83 & 0,62 & 0,84 \\
\hline STM1106 & 0,74 & 0,74 & 0,00 & 0,74 & 0,58 & 0,82 & 0,17 & 0,7 \\
\hline STM5114 & 0,72 & 0,66 & 0,59 & 0,69 & 0,78 & 0,7 & 0,64 & 0,74 \\
\hline STM5127 & 0,79 & 0,7 & - & 0,75 & 0,82 & 0,78 & 0,59 & 0,8 \\
\hline STPoAc58 & 0,55 & 0,51 & 0,35 & 0,53 & 0,62 & 0,56 & 0,65 & 0,59 \\
\hline Promedio & 0,66 & 0,57 & 0,37 & & 0,68 & 0,64 & 0,52 & \\
\hline
\end{tabular}

*SSR: Simple Sequence Repeats (marcador microsatélite), ADG: Solanum tuberosum subsp. andigena; STN: Solanum stenotomum, JUZ Solanum x juzepczukii. Los valores de PIC enmarcados son los mayores para cada marcador y especie.

Analizando valores promedios, la especie S. tuberosum subsp. andigena presentó el mayor valor de PIC con 0,66 seguido por S. stenotomum con 0,57 y $S$. x juzepczukii con 0,37 . Comparando el valor promedio de polimorfismo de cada microsatélite, se observó que los microsatélites STM1104, STG0016, STI0030, STG0010 y STM0037 fueron los más polimórficos. Estos resultados son comparables a los reportados por Cadima et al. (2013) para la colección nacional, y por Ghislain et al. (2009) en la colección del CIP.

Por lo tanto, estos datos confirman la representatividad de las especies $S$. tuberosum subsp. andigena y $S$. stenotomum en Colomi.

\section{Caracterización de la diversidad genética de papa de Transecto del Lago (Cachilaya y Coromata)}

Al comparar la colección de papa del transecto del Lago y la colección nacional, se observa una buena representatividad en las especies $S$. tuberosum subsp. andigena y $S$. stenotonum por no existir diferencias

significativas entre las papas de ambas colecciones en la mayoría de las características morfológicas evaluadas (Tabla 8). 
Tabla 8. Significancia estadística de la comparación de medias y varianzas entre las papas del transecto del Lago y la colección nacional

\begin{tabular}{|c|c|c|c|c|c|c|c|c|}
\hline \multirow{3}{*}{$\begin{array}{c}\text { Caracteres } \\
\text { morfológicos }\end{array}$} & \multicolumn{4}{|c|}{ Cachilaya } & \multicolumn{4}{|c|}{ Coromata } \\
\hline & \multicolumn{2}{|r|}{ ADG } & \multicolumn{2}{|r|}{ STN } & \multicolumn{2}{|c|}{ ADG } & \multicolumn{2}{|c|}{ STN } \\
\hline & Media & Varianza & Media & Varianza & Media & Varianza & Media & Varianza \\
\hline $1 \mathrm{CBY}$ & n.s. & n.s. & n.s. & $* *$ & & & & \\
\hline 2 CLZ & n.s. & n.s. & $* *$ & n.s. & & & & \\
\hline $3 \mathrm{CPF}$ & n.s. & n.s. & n.s. & n.s. & & & & \\
\hline $4 \mathrm{CPP}$ & $* *$ & n.s. & $* *$ & n.s. & & & & \\
\hline $5 \mathrm{CPPL}$ & n.s. & n.s. & n.s. & n.s. & n.s. & n.s. & n.s. & n.s. \\
\hline $6 \mathrm{CSF}$ & $* *$ & n.s. & $* *$ & $* *$ & & & & \\
\hline 7 CSP & n.s. & n.s. & n.s. & n.s. & n.s. & n.s. & n.s. & n.s. \\
\hline 8 CSPL & n.s. & n.s. & n.s. & n.s. & & & & \\
\hline $9 \mathrm{CTL}$ & n.s. & n.s. & n.s. & n.s. & & & & \\
\hline 10 DCS & $* *$ & n.s. & $* *$ & n.s. & & & & \\
\hline 11 DCSP & n.s. & n.s. & n.s. & $* *$ & & & & \\
\hline 12 DSCPL & n.s. & n.s. & n.s. & n.s. & n.s. & n.s. & n.s. & n.s. \\
\hline $13 \mathrm{FBY}$ & $* *$ & $* *$ & n.s. & n.s. & & & & \\
\hline 14 FCL & *** & n.s. & n.s. & $* *$ & & & & \\
\hline 15 FGR & n.s. & n.s. & $* *$ & n.s. & $* *$ & n.s. & ** & n.s. \\
\hline 16 FRA & n.s. & $* *$ & n.s. & n.s. & n.s. & n.s. & n.s. & $* *$ \\
\hline 17 GFL & n.s. & $* *$ & n.s. & n.s. & & & & \\
\hline $18 \mathrm{HPL}$ & n.s. & $* *$ & n.s. & n.s. & n.s. & n.s. & n.s. & $* *$ \\
\hline 19 INT & n.s. & n.s. & n.s. & n.s. & & & & \\
\hline 20 INTC & $* *$ & $* *$ & n.s. & n.s. & n.s. & n.s. & n.s. & n.s. \\
\hline $21 \mathrm{POJ}$ & $* *$ & n.s. & $* *$ & n.s. & n.s. & n.s. & n.s. & n.s. \\
\hline 22 TDS & n.s. & n.s. & n.s. & n.s. & n.s. & n.s. & n.s. & n.s. \\
\hline
\end{tabular}

${ }^{1}$ Ver Tabla 3 para el significado de las siglas de los caracteres morfológicos $\mathrm{ns}: \mathrm{P}>0,05 ; * *: \mathrm{P}<0,01 ;$ ADG: S. tuberosum subsp. andigena; STN: S. stenotonum

\section{Caracterización de la diversidad genética de papa del Altiplano Norte}

Comparando la colección de papa del Altiplano Norte y la colección nacional se observa una buena representatividad en las especies $S$. stenotonum, $S$. goniocalyx y $S$. $x$ curtilobum por no existir diferencias significativas entre las papas de ambas colecciones en la mayor parte de las características morfológicas evaluadas (Tabla 9), mientras que en $S$. tuberosum subsp. andigena, $S$. $x$ ajanhuiri y en $S . x$ juzepczukii el número de variables con diferencias significativas es mayor cuando se compara las medias, pero el número de variables que no son significativas sigue

siendo mayor cuando se comparan las varianzas. Las diferencias significativas en $S$. tuberosum subsp. andigena más importantes se dan en los colores secundarios de la flor, de la piel del tubérculo y de la pulpa, así como en la distribución de estos colores secundarios. Hay también diferencia significativa en la forma del tubérculo, la forma de la corola y el hábito de crecimiento de la planta. Los tubérculo son especialmente resaltantes porque es el carácter que contribuye más en la diferenciación de cultivares y 
especies, y son los caracteres de mayor uso por los agricultores (Terrazas et al., 2005;

Raime \& Checya s/f). 
Tabla 9. Significancia estadística de la comparación de medias y varianzas entre las papas del Altiplano Norte y la colección nacional

\begin{tabular}{|c|c|c|c|c|c|c|c|c|c|c|c|c|}
\hline \multirow{2}{*}{$\begin{array}{l}\text { Caracteres } \\
\text { morfológicos }\end{array}$} & \multicolumn{2}{|c|}{ ADG } & \multicolumn{2}{|c|}{ STN } & \multicolumn{2}{|c|}{ GON } & \multicolumn{2}{|c|}{ АЈH } & \multicolumn{2}{|c|}{ JUZ } & \multicolumn{2}{|c|}{ CUR } \\
\hline & Media & Varianza & Media & Varianza & Media & Varianza & Media & Varianza & Media & Varianza & Media & Varianza \\
\hline $\mathrm{CPF}$ & n.s. & n.s. & $* *$ & n.s. & $* *$ & n.s. & $* *$ & n.s. & $* *$ & $* *$ & $* *$ & $* *$ \\
\hline CPP & n.s. & $* *$ & n.s. & n.s. & n.s. & n.s. & $* *$ & n.s. & n.s. & $* *$ & n.s. & n.s. \\
\hline CPPL & n.s. & n.s. & n.s. & n.s. & n.s. & n.s. & n.s. & n.s. & n.s. & n.s. & n.s. & n.s. \\
\hline $\mathrm{CSF}$ & $* *$ & n.s. & $* *$ & n.s. & n.s. & $* *$ & $* *$ & $* *$ & $* *$ & $* *$ & $* *$ & n.s. \\
\hline CSP & $* *$ & n.s. & n.s. & n.s. & n.s. & n.s. & n.s. & $* *$ & $* *$ & $* *$ & n.s. & $* *$ \\
\hline CSPL & $* *$ & $* *$ & n.s. & n.s. & n.s. & n.s. & n.s. & n.s. & n.s. & n.s. & n.s. & n.s. \\
\hline CTL & n.s. & n.s. & n.s. & n.s. & n.s. & n.s. & n.s. & n.s. & $* *$ & $* *$ & n.s. & n.s. \\
\hline DCS & $* *$ & n.s. & $* *$ & n.s. & $* *$ & n.s. & $* *$ & $* *$ & $* *$ & $* *$ & $* *$ & n.s. \\
\hline DCSP & $* *$ & $* *$ & n.s. & n.s. & n.s. & n.s. & n.s. & $* *$ & n.s. & n.s. & n.s. & n.s. \\
\hline DSCPL & $* *$ & n.s. & n.s. & n.s. & n.s. & n.s. & n.s. & n.s. & $* *$ & n.s. & n.s. & n.s. \\
\hline FCL & $* *$ & $* *$ & $* *$ & n.s. & n.s. & n.s. & n.s. & n.s. & $* *$ & $* *$ & & \\
\hline FGR & $* *$ & n.s. & n.s. & n.s. & n.s. & n.s. & n.s. & n.s. & $* *$ & n.s. & n.s. & n.s. \\
\hline FRA & n.s. & n.s. & n.s. & n.s. & n.s. & n.s. & $* *$ & n.s. & n.s. & n.s. & n.s. & $* *$ \\
\hline GFL & n.s. & n.s. & n.s. & n.s. & n.s. & n.s. & n.s. & n.s. & $* *$ & n.s. & & \\
\hline HPL & $* *$ & n.s. & $* *$ & n.s. & n.s. & n.s. & $* *$ & n.s. & $* *$ & $* *$ & $* *$ & n.s. \\
\hline INT & $* *$ & $* *$ & $* *$ & n.s. & $* *$ & n.s. & $* *$ & n.s. & $* *$ & n.s. & $* *$ & n.s. \\
\hline INTC & $* *$ & n.s. & n.s. & n.s. & $* *$ & n.s. & $* *$ & n.s. & $* *$ & n.s. & $* *$ & n.s. \\
\hline POJ & $* *$ & $* *$ & n.s. & $* *$ & n.s. & n.s. & $* *$ & $* *$ & n.s. & n.s. & n.s. & n.s. \\
\hline TDS & n.s. & n.s. & n.s. & n.s. & n.s. & n.s. & n.s. & n.s. & n.s. & n.s. & n.s. & n.s. \\
\hline
\end{tabular}

${ }^{1}$ Ver Tabla 3 para el significado de las siglas de los caracteres morfológicos; ns : P > 0,05; ** : P < 0,01; ADG: S. tuberosum subsp. andigena; STN: S. stenotonum; GON: $S$. goniocalyx; AJH: $S . \quad x$ ajanhuiri; JUZ: $S . \quad x$ juzepczukii; CUR: $S . \quad x$ curtilobum. 
En $\quad S . \quad x$ ajanhuiri las diferencias al comparar las medias más importantes se dan en la flor y el tubérculo. En $S$. $x$ juzepczukii las diferencias en medias más importantes se observan en el color secundario de la flor y de la piel del tubérculo, y también en la forma general del tubérculo y el hábito de crecimiento de la planta.

Esta información sugiere que la representación de la diversidad de $S$. tuberosum subsp. andigena y $S$. $x$ juzepczukii en el Altiplano Norte es importante por el número de cultivares encontrados de estas dos especies en esta región, pero la riqueza de la diversidad representa parcialmente a lo existente en la colección nacional. En el caso de $S$. $x$ ajanhuiri el número de cultivares de esta especie en el Altiplano norte no es representativo de la diversidad de la colección nacional.

\section{Implicancias en la conservación in situ de la diversidad de papas nativas}

La información sobre la diversidad encontrada en papa por sus caracteres morfológicos y moleculares en los cuatro centros de agrobiodiversidad objeto de este estudio, y la comparación realizada con la colección nacional, provee información valiosa y útil para entender, al menos parcialmente, cómo los agricultores y comunidades en estos centros están manejando la diversidad genética de este cultivo. Por un lado, nos indican que hay una diversidad importante existente a nivel in situ particularmente para las especies $S$. tuberosum subsp. andigena y $S$. stenotonum, las cuales están ampliamente representadas en los cuatro centros de agrobiodiversidad. En cambio la diversidad encontrada en las otras especies, $S . x$ ajanhuiri, S. x juzepczukii, S. goniocalyx y S. $x$ curtilobum, representó parcialmente lo que existente en la colección nacional. Aunque el estudio demuestra la existencia de diversidad importante a nivel in situ, aún no es suficiente para estimar el estado de conservación de esta diversidad, porque no fue posible determinar por ejemplo cuántos agricultores $\mathrm{o}$ en qué superficie (número de chacras) son cultivadas las diferentes especies y sus cultivares. Se entiende que a mayor número de agricultores que mantengan un cultivar, mejora las probabilidades de su conservación (Jarvis et al., 2016; Baena et al., 2003).

\section{Conclusiones}

La información proporcionada por los análisis moleculares con marcadores microsatélites y análisis morfológicos permitieron establecer la representatividad genética de las colecciones de papa existentes en centros de agrobiodiversidad. Se sugiere sin embargo que para futuros estudios similares la aplicación de solo análisis molecular porque no son influenciados por factores externos $\mathrm{y}$ medioambientales durante la generación de la información. Podrían explorarse otros marcadores de última generación como los SNP (Single Nucleotide Polymorphism) que son más abundantes, estables y pueden revelar polimorfismo no detectados por otros marcadores (Ravi et al., 2014).

Se confirma que la especie Solanum tuberosum subsp. andigena es la especie con mayor diversidad genética tanto a nivel molecular como morfológico en los centros de agrobiodiversidad, seguida de $S$. stenotomum.

Se evidencia también una menor diversidad en las especies $S$. $x$ ajanhuiri, $S$. $x$ juzepczukii, $S$. goniocalyx y $S . \quad x$ curtilobum, encontrándose pocos cultivares particularmente de las tres últimas especies en los centros de agrobiodiversidad estudiados.

Los datos moleculares muestran que la diversidad genética de papa del centro de agrobiodiversidad del Norte Potosí es representativa de la diversidad total de la colección nacional para las especies $S$. tuberosum subsp. andigena, $S$. stenotomum, $S . x$ juzepczukii y $S . \quad x$ 
ajanhuiri, debido a que más del $60 \%$ de los alelos de la colección nacional se hallan representados en este centro de agrobiodiversidad.

En Colomi se ha encontrado una representatividad importante para las especies $S$. tuberosum subsp. andigena y $S$. stenotomum, que a pesar de haberse hecho el estudio en un número reducido de variedades respecto a los otros centros de agrobiodiversidad, se ha hallado al menos el $50 \%$ de alelos de la colección nacional representados en Colomi para estas dos especies.

Los datos morfológicos muestran que la diversidad genética de papa del transecto del lago (Cachilaya y Coromata) es representativa de la diversidad de la colección nacional para las especies $S$. tuberosum subsp. andigena y $S$. stenotomum, debido a que no se encontraron diferencias significativas en más del $70 \%$ de las variables morfológicas consideradas en este estudio.

En las papas del Altiplano norte, la diversidad de S. stenotonum, S. goniocalyx y $\quad S . \quad x$ curtilobum representa significativamente lo existente en la colección nacional para estas especies. Las papas de $S$. tuberosum subsp. andigena y $S$. $x$ juzepczukii contienen también una importante variabilidad pero es parcial de lo existente en la colección nacional. Las papas de S. $x$ ajanhuiri encontradas en este centro de agrobiodiversidad no representan la variabilidad de la colección nacional.

Los centros de agrobiodiversidad mantienen una diversidad importante de papa cultivada y justifican su valor como conservadores y dinamizadores de la variabilidad genética de este cultivo.

Resalta la importancia de la conservación de la colección nacional para resguardar la diversidad genética de papa y también para reponer diversidad en las comunidades que así lo requieran.

\section{Conflictos de intereses}

No se tiene ningún conflicto de intereses con ningún sector.

\section{Literatura citada}

Aranguren-Méndez, J.A.; Román-Bravo, R.; Isea, W.; Villasmil, Y.; Jordana, J. 2005. Los microsatélites (STR's), marcadores moleculares de ADN por excelencia para programas de conservación. Archivos Latinoamericanos de Producción Animal 1(13): 30-42.

Baena, M.; Jaramillo, S.; Montoya, J.E. 2003. Material de apoyo a la capacitación en conservación in situ de la diversidad vegetal en áreas protegidas y en fincas. Instituo Internacional de Recursos Fitogenéticos, Cali, Colombia. 130p.

Botstein, D.; White, R.L.; Skolnick, M.; Davis, R.W. 1980. Construction of a genetic linkage map in man using restriction fragment length polymorphisms. Am J Hum Gen. 32:314-331.

Cadima, X.; Veramendi, S.; Gabriel, J. 2013. Uso de marcadores moleculares microsatélite para el análisis de la diversidad genética de papa nativa de Bolivia. Journal of the Selva Andina Research Society 4(1): 1-14.

Cadima, X.; Gonzáles, R.; Almanza, J.; García, W.; Terrazas, F. (eds). 2004. Catálogo de variedades locales de papa y oca de la zona de Candelaria. Serie: Conservación y uso de la biodiversidad de raíces y tubérculos andinos: Una década de investigación para el desarrollo (19932003). No. 5. Fundación PROINPA, Municipio de Colomi, CIP, COSUDE. Cochabamba, Bolivia. 113 p.

Castillo J.A.; Plata G. 2016. The expansion of brown rot disease throughout Bolivia: possible role of climate change. Can. J. Microbiol. 62: 1-7 dx.doi.org/10.1139/cjm-2015-0665. 
de Haan, S.; Nuñez, J.; Bonierbale, M.; Ghislain, M. 2010. Multilevel agrobiodiversity and conservation of Andean potatoes in Central Peru: Species, morphological, genetic, and spatial diversity. Moun Res Develop 30: 222-231.

de Vicente, M.C.; Fulton, T. (eds.). 2004. Tecnologías de Marcadores Moleculares para Estudios de Diversidad Genética de Plantas: Módulo de Aprendizaje. Vol 1. Illus. Nelly Giraldo. Instituto Internacional de Recursos Fitogenéticos (IPGRI), Roma, Italia.

Doyle, J.J.; Doyle, J.L. 1990. Isolation of plant DNA from fresh tissue. BRL Focus. 12: 13-15.

Eyzaguirre-Rodríguez, J.L. 2015. Importancia socioeconómico de la agricultura familiar en Bolivia. TIERRA, La Paz, Bolivia. 92 p.

FAO, 2017a. El futuro de la alimentación y la agricultura: tendencias y desafíos. Disponible en www.fao.org/3/a-i6583e.pdf (consultado el 13/04/2017).

FAO, 2017b. Recursos fitogenéticos, o se utilizan $o$ se pierde. Disponible en http://www.fao.org/fileadmin/templates/nr/ documents/CGRFA/factsheets_plant_es.pd f (consultado el 13/04/2017).

Franco, T.L.; R. Hidalgo (eds.). 2003. Análisis Estadístico de Datos de Caracterización Morfológica de Recursos Fitogenéticos- Boletín técnico $\mathrm{N}^{\circ} 8$, Instituto Internacional de Recursos Fitogenéticos (IPGRI), Cai, Colombia. 89 p.

Ghislain, M.; Nuñez, J.; Herrera, M.dR.; Pignataro, J.; Guzmán, F.; Bonierbale, M.; Spooner, D.M. 2009. Robust and highly informative microsatellite-based genetic identity kit for potato. Mol Breed. 23: 377388

Gómez, R. 2000. Guía para las caracterizaciones morfológicas báscias en colecciones de papa. International Potato Center (CIP), Lima - Perú.
Hawkes, J.G.; Hjerting, J.P. 1989. The potatoes of Bolivia: Their breeding and evolutionary relationships. Oxford University Press. Oxford, UK. 472 p.

He, C.; Poysa, V.; Yu, K. 2003. Development and characterization of simple sequence repeat (SSR) markers and their use in determining relationships among Lycopersicon esculentum cultivars, Theoretical and Applied Genetics 106 (2): 363-373.

Iriarte, V.; Condori, B.; Parapo, D.; Acuña, D. 2009. Catálogo etnobotánico de papas nativas del Altiplano Norte de La PazBolivia. Fundación PROINPA, Ricerca e Cooperzione; Instituto Nacional de Innovación Agropecuaria y Forestal. Cochabamba, Bolivia. 142 p.

Jarvis, D.I.; Hodgkin, T.; Brown, A.H.D.; Tuxil, J.; López-Noriega, I.; Smale, M.; Sthapit, B. 2016. Crop Genetic Diversity in the Field and on the Farm: Principles and Applications in Research Practices. Yale University Press, New Haven \& London. $395 \mathrm{p}$.

Khoury, C.K.; Achicanoy, H.A.; Bjorkman, A.D.; Navarro-Racines, C.; Guarino, L.; Flores-Palacios, X.; Engels, J. M. M.; Wiersema, J. H.; Dempewolf, H.; Sotelo, S.; Ramírez-Villegas, J.; Castañeda-Alvarez, N. P.; Fowler, C.; Jarvis, A.; Rieseberg, L. H.; Struik, P. C. 2016. Origins of food crops connect countries worldwide. Proc. R. Soc. B 283: 20160792.

http://dx.doi.org/10.1098/rspb.2016.0792

Krapovickas, A. 2010. La domesticación y el origen de la agricultura. BONPLANDIA 19(2): 193-199.

Martin, K.; Sauerborn, J. 2013. Agroecology. Springer Dordrecht Heidelberg New York London. 330 p.

Ochoa, C. 1990. The Potatoes of South America: Bolivia. Cambridge University Press, Cambridge, UK. 535 p. 
Pérez, J.R. 2004. Evaluación de la diversidad genética de papas nativas (Solanum tuberosum L. ssp. tuberosum Hawkes) silvestres y cultivadas del sur de Chile, mediante el uso de marcadores microsatélites. Tesis de licenciatura en Agronomia. Universidad Austral de Chile, Valdivia-Chile. 68 p.

Raime, L.; Checya, D. s/f. Conservación in situ de la agrobiodiversidad andinoamazónica. Sistematización sobre caracterización campesina de variedades de papa en comunidades conservacionistas de Paucartambo. Proyecto Conservación in situ de cultivos nativos y sus parientes silvestres. PER/98/G33. PNUD-Perú. Centro de Servicios Agropecuarios. $51 \mathrm{p}$.

Ravi, I.; Baunthiyal, M.; Saxena, J. (eds.) 2014. Advances in Biotechnology. Springer New Delhi Heidelberg New York Dordrecht London.

Rohlf, F.J. 2004. NTSYS-pc: Numerical Taxonomy and Multivariate Analysis System, Version 2.1 Exeter Software, Setauket, New York.

Snedecor, G. W.; Cochran, W. G. 1989. Statistical Methods. Eighth Edition, Iowa State University Press. 503 p.
Terrazas, F.; Cadima, X.; García, R.; Zeballos, J. 2008. Catálogo etnobotánico de papas nativas. Tradición y cultura de los Ayllus del Norte Potosí y Oruro. Ricerca \& Cooperazione, Centro de Apoyo al Desarrollo, GTZ, Fundación PROINPA, Ministerio de Desarrollo Rural, Agropecuario y Medio Ambiente. Cochabamba - Bolivia. 189 p.

Terrazas, F; Guidi, A; Cadima, X.; Gonzales, R.; Chávez, E.; Almanza, J.; Salazar, M.; Baudoin, J.P. 2005. Conservación in situ y valoración de las papas nativas en el microcentro de diversidad genética de Candelaria, Cochabamba-Bolivia. Agrociencia 9(1): 135-146.

Torrez, M.E.; Moreno, S. 2001. Caracterización mediante marcadores moleculares basados en ADN. pp 235 253. En: Gonzáles-Andrés, F.; PitaVillamil, J. (eds.). Conservación y caracterización de recursos fitogenéticos. Primera edición. Escuela Universitaria de Ingeniería Técnica Agrícola I.N.E.A., Valladolid, España. 279 p. 